\title{
DEFORMATION BEHAVIOUR OF MG-BASED MATRIX COMPOSITES
}

The influence of various reinforcements on mechanical properties and deformation behaviour of some magnesium alloys was studied. Strength and deformation behaviour were investigated over a wide temperature range. The reinforcement was in the form of short fibres or particles. The volume fraction of the reinforcement significantly influences the properties of a composite. The interface between the matrix and the reinforcement has a great deal of importance in determining the properties of the composite. Thermal stresses generated at the interface play a very important role.

\section{Introduction}

Magnesium, the lightest structural metallic element, and its alloys possess a great potential to be developed as structural materials provided that significant improvements in properties are achieved. Coarse grained $\mathrm{Mg}$ materials have some limitations such as low strength, low ductility and poor corrosion resistance. It can be shown that grain size refinement is an effective method for improving the strength of materials. According to the Hall-Petch relationship, magnesium alloys with ultrafine grains have high specific strength and specific stiffness. Grain refinement leads, in some cases, to a higher ductility of the magnesium alloys. On the other hand, Mg alloys exhibit poor plastic formability due to their hexagonal close packed structure.

Reinforcement of an alloy leads also to an increase in strength [1]. Magnesium alloys are attractive candidates for reinforcement with short ceramic fibres or particles. Reinforcement allows adapting more exactly the work piece material properties to requirements. Therefore, there is an increasing trend in industry branches to use the metallic matrix composites (MMC) for various parts. Thermal residual stresses are formed in MMCs due to differences in the coefficients of thermal expansion between the matrix and the reinforcement. The matrix/reinforcement interface is significant in determining mechanical properties. In general, composite properties strongly depend on the properties of the composite components. The interface region is the region where a discontinuity in some material parameters occurs.

The aim of this paper is to show the influence of temperature on the strength and deformation behaviour of some magnesium based MMCs.

\section{Experimental procedure}

The investigated AZ91 magnesium alloy reinforced with $\delta$ $\mathrm{Al}_{2} \mathrm{O}_{3}$ short fibres (Saffil ${ }^{\circledR}$ with a mean diameter of $3 \mu \mathrm{m}$ and a mean length about $87 \mu \mathrm{m}$ ) was prepared by squeeze casting technology. The preforms consisting of $\mathrm{Al}_{2} \mathrm{O}_{3}$ short fibres showing a planar isotropic distribution and a binder system (containing $\mathrm{Al}_{2} \mathrm{O}_{3}$ and starch) were preheated to a temperature (about $1000^{\circ} \mathrm{C}$ ) higher than the melt temperature of magnesium and then inserted into preheated die $\left(290\right.$ to $\left.360{ }^{\circ} \mathrm{C}\right)$. The pressure for forcing the melt into the die with the preform was applied in two steps (e.g. $50 \mathrm{MPa}$ for $10 \mathrm{~s}$ and $130 \mathrm{MPa}$ for $60 \mathrm{~s}$; the values of the pressure depend on the alloy used). The second step closes pores and shrinkage cavities. During this short time of contact between the liquid metal and the fibres, only a slight reaction between the fibres and the matrix can occur.

The WE54 alloy reinforced with $\mathrm{SiC}$ particles (a volume fraction of $13 \%$ ) was processed by a powder metallurgy method. Mixing of the matrix powders with various $\mathrm{SiC}$ particles was carried out first in an asymmetrically moved mixer with subsequent milling in a ball mill. The powder was capsulated in magnesium containers and extruded at $400{ }^{\circ} \mathrm{C}$ using a $400 \mathrm{t}$ horizontal extrusion press. The composite samples were not thermally treated. The mean SiC particle size was found to be about $9 \mu \mathrm{m}$. The as-extruded bar appeared mainly constituted of very small equiaxed grains (about $3 \mu \mathrm{m}$ ) after accurate optical microscopy observations. No grain growth was observed during the deformation tests at elevated temperatures.

The mechanical properties of the composites were assessed through compression tests at temperatures between 20 and $300{ }^{\circ} \mathrm{C}$.

\footnotetext{
* Pavel Lukac, Zuzanka Trojanova

Charles University, Department of Physics of Materials, Prague, Czech Republic,

E-mail: lukac@met.mff.cuni.cz
} 
The specimens with dimensions $5 \times 5 \times 10 \mathrm{~mm}^{3}$ were deformed using an Instron testing machine at a constant crosshead speed giving an initial strain rate $2.7 \times 10^{-4} \mathrm{~s}^{-1}$.

\section{Experimental results and discussion}

Short fibres in the composites were randomly arranged (a 2D random orientation), i.e. axes of the fibres lay in parallel planes. Specimens of AZ91 alloys reinforced with 23 vol.\% short Saffil fibres were prepared with both the fibre planes parallel to and perpendicular to the specimen axis. The temperature dependences of the yield stress of both monolithic AZ91alloy and reinforced with 23 vol. \% Saffil fibres with both fibre planes orientations are given in Fig. 1. The maximum stress for the same specimens as a function of the test temperature is given in Fig. 2.

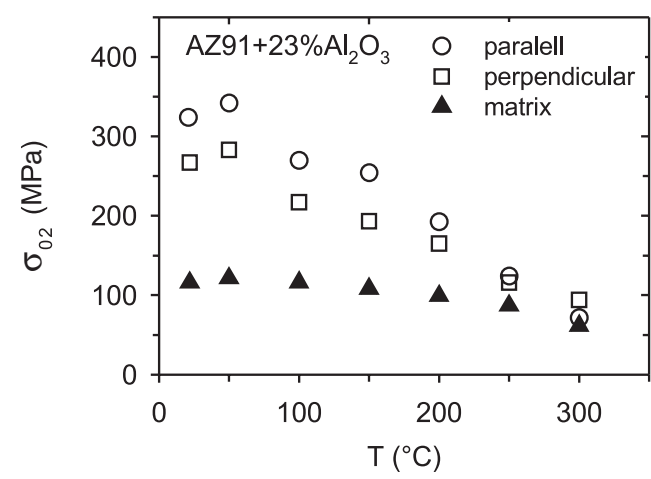

Fig. 1 Temperature dependence of yield stress for AZ91 and $\mathrm{AZ91}+\mathrm{Al}_{2} \mathrm{O}_{3}$

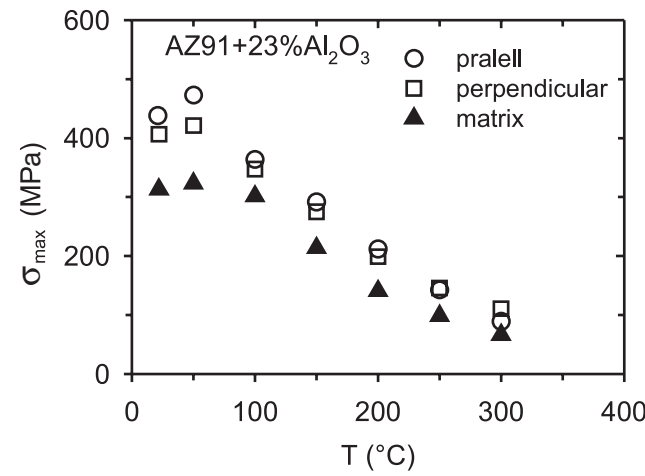

Fig. 2 Temperature dependence of maximum stress for AZ91 and $\mathrm{AZ91}+\mathrm{Al}_{2} \mathrm{O}_{3}$

The yield stress and the maximum stress decrease very rapidly with increasing temperature. At and above $250^{\circ} \mathrm{C}$ the values of the yield stress for the composite are very close to those for the monolithic alloy (matrix). It is clear the yield stress of the AZ91 composite at lower temperatures (below $200{ }^{\circ} \mathrm{C}$ ) is higher than that of unreinforced alloy. The difference between the yield stress of the composite and that of monolithic alloy at room temperature is more than $200 \mathrm{MPa}$. On the other hand, the values of the maximum stress for the composites are very close to those for the unreinforced alloy. This may indicate that fibres influence significantly the yield and that dislocation motion is more important for strain hardening. It should be mentioned that the difference between the maximum stress and yield stress decreases with increasing temperature. This indicates a softening process at higher temperatures. The temperature dependences of the characteristic stresses of WE54+SiC composite, the yield stress $\sigma_{02}$ and the maximum stress $\sigma_{\max }$, are shown in Fig. 3.

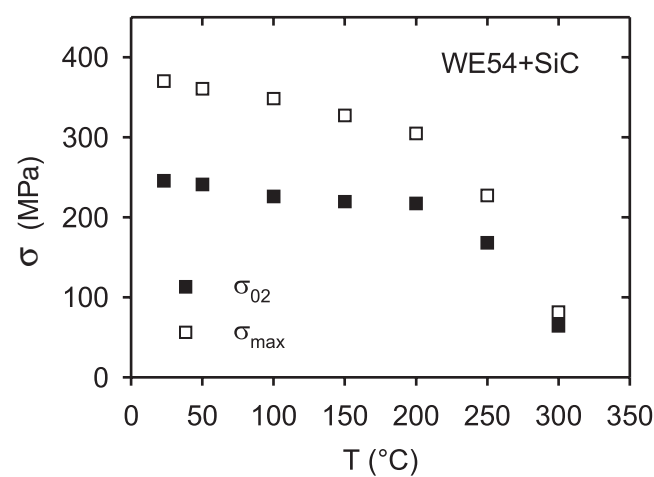

Fig. 3 Temperature dependence of yield stress and maximum stress for $\mathrm{WE} 54+\mathrm{SiC}$

The influence of the temperature on strain hardening of the composite is well visible. The yield stress decreases with increasing temperature very slowly up to $200{ }^{\circ} \mathrm{C}$, while the observed decrease of the maximum stress omax is more rapid. For temperatures higher than $200{ }^{\circ} \mathrm{C}$ both stresses decrease substantially. It can be concluded that the thermal stability of the composite is very good up to $200{ }^{\circ} \mathrm{C}$. TEM micrograph of the non-deformed WE54+SiC composite sample is shown in Fig. 4.

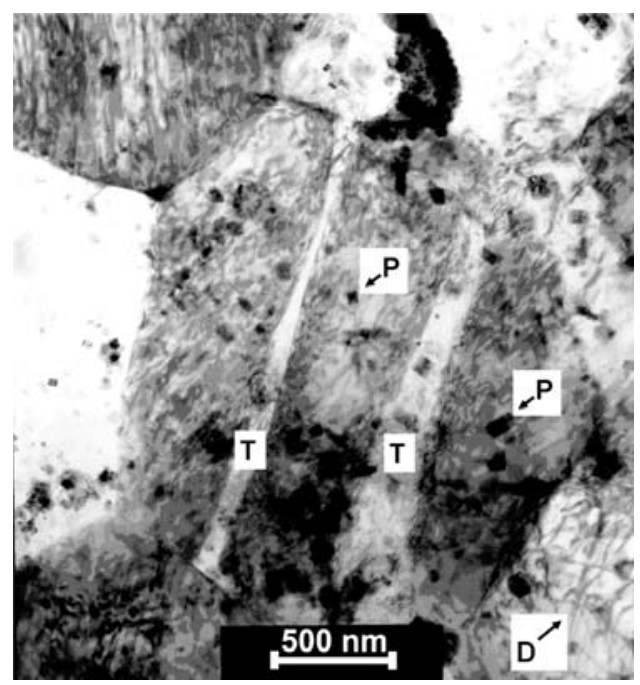

Fig. 4 TEM micrograph of WE54+SiC composite 
TEM investigation revealed the presence of many twins (T) and precipitates $(\mathrm{P})$. Tangled dislocations (D) in the right bottom corner are visible. The presence of twins is a common feature for both non-deformed as well as deformed composites. Grains in the as-received material are well visible in Fig. 4. Thin twins, within single grains, are parallel to each other and are quite narrow. Sometimes twins are extended trough the grain boundary, causing a grain boundary deflection.

It can be seen that addition of the reinforcing phase substantially increases the yield stress. Generally, fibres in the matrix act as obstacles for dislocation motion in the slip plane. Accordingly, the stress necessary for plastic deformation of composites should be higher than in the unreinforced matrix. The load transfer and enhanced dislocation density should be considered as significant factors influencing the value of the yield stress.

\subsection{Yield stress}

The load transfer from matrix to fibre is maintained by the interface. According to the shear lag theory the load transfer occurs between reinforcement with a high aspect ratio and the matrix by means of the shear stress at the fibre-matrix interface. In this mechanism, the fibre can act as a "reinforcement" to carry some of the load. A contribution to the yield stress due to load transfer $\Delta \sigma_{L T}$ is given, according to shear lag model [2], as

$$
\Delta \sigma_{L T}=\sigma_{m}\left[1+\frac{(L+t) A}{4 L}\right] f+\sigma_{m}(1-f)
$$

where $\sigma_{m}$ is the yield stress of the matrix, $L$ is the fibre size in the applied stress direction, $t$ is the fibre size in the perpendicular direction and $A$ is the fibre aspect ratio $(L / t)$. For equiaxial particles, an increase in the yield stress due to load transfer $\Delta \sigma_{L T}$ may be given by

$$
\Delta \sigma_{L T}=\sigma_{m} 0.5 f
$$

The strengthening component $\Delta \sigma_{L T}$, depends on the reinforcement volume fraction.

The strengthening component $\Delta \sigma_{L T}$ in AZ91 - 23 vol. \% Saffil fibres, arising from the load transfer form matrix to fibres, has a value of $180 \mathrm{MPa}$ in the case when all fibres are aligned in the specimen axis. Calculating the strength increase of 2D randomly oriented short fibres, one becomes $\Delta \sigma_{L T}=108 \mathrm{MPa}$.

Typically, there is a large difference in the values of therma expansion coefficients (CTE) between the matrix and the fibres. During the fabrication process of a composite and/or during heat treatment, thermal stresses are generated owing to different therma expansion of the matrix and the reinforcement. For a composite with short fibres, the thermal stresses $\sigma_{T S}$ generated at the vicinity of the matrix-reinforcement interface can be calculated as [3, 4]

$$
\sigma_{T S}=E_{f} E_{M} f \Delta \alpha \Delta T /\left[E_{f} f+E_{M}(1-f)\right] .
$$

Here $E_{f}$ and $E_{M}$ are the values of Young's modulus for the fibres and the matrix, respectively, $f$ is the volume fraction of fibres, $\Delta \alpha$ is the difference of the CTE values and $\Delta T$ is the temperature change from the free-stress state. With increasing distance from the fibres, the thermal stress is lower because it decreases with the distance $r$ as $1 / r^{3}$. Volume-averaged residual stresses reach in the matrix their maximum value [5]

$$
\sigma_{m \max }=\frac{2}{3} \sigma_{y} \ln \left(\frac{1}{f}\right) 1-f
$$

where $\sigma y$ is the yield stress in the matrix. The thermal stresses in the matrix may exceed the yield stress. Then plastic deformation occurs without any applied stress and new dislocations are formed at the interfaces. The dislocation density in the vicinity of the interfaces was found to be significantly higher than that elsewhere in the matrix. Plastic zones containing tangled dislocations generally result along the fibres [6]. The density of the newly created dislocations, $\Delta \rho$, is given as [4, 7]

$$
\Delta \rho=\frac{B f \Delta \alpha \Delta T 1}{b(1-f) t_{f}}
$$

where $t_{f}$ is the minimum size of the reinforcing phase, $b$ is the magnitude of the Burgers vector of dislocations and $B$ is a geometrical constant depending on the reinforcement shape.

The generation of thermally induced dislocations and the related dislocations density gradients increase also the yield stress of the composite. A contribution to the yield stress due to the increase in the dislocation density may be expressed as

$$
\sigma_{C T E}=\alpha_{1} \psi G b\left(\frac{B f \Delta \alpha \Delta T}{(1-f) b t_{f}}\right)^{\frac{1}{2}},
$$

where $\alpha_{1}$ is a constant, $G$ the shear modulus and $\psi$ is the Taylor factor. The density of the new dislocations formed during preparation process is approximately $\Delta \rho=1.87 \times 1013 \mathrm{~m}^{-2}$ (if $B=10$, $\left.\Delta \alpha=20 \times 10^{-6} \mathrm{~K}^{-1}\right)$. The stress contribution at room temperature $\Delta \sigma_{C T E}$ is about $49 \mathrm{MPa}$, if using (6) and taking $\alpha_{1}=0.35$ and Taylor factor $\psi=6$ [8].

As another mechanism contributing to the yield stress one should consider the generation of geometrically necessary dislocations [9], the density of which is, in the yield stress vicinity, about $3.8 \times 1.012 \mathrm{~m}^{-2}$. The corresponding stress increase is about $22 \mathrm{MPa}$. Very probably this mechanism is not significant at the yield stress but the density of the geometrically necessary dislocations increases with increasing strain. However, it is important to note that the discontinuously reinforced composites (with particles) have very fine grains; smaller than their unreinforced matrixes. The contribution to the yield stress due to this effect can then be estimated using the Hall-Petch relation.

Fibres or particles in the matrix are obstacles for dislocation motion. Therefore, the stress necessary for the motion of dislocations in the composite is higher than in the matrix without fibres. 
We may consider a similar mechanism as in the case of strengthening by incoherent particles, i.e. Orowan strengthening. It is caused by the resistance of closely spaced hard fibres to the passing of dislocations. Asides from fibres, dislocations overcome stresses from dislocation pile-ups in the vicinity of the fibres. For strengthening caused by this mechanism, one may use to the following equation $[10]$

$$
\Delta \sigma_{O R}=\frac{G b}{\Lambda}+\frac{5}{2 \pi} G f \varepsilon_{p}
$$

where $\Lambda$ is the distance between fibres and $\varepsilon_{p}$ is plastic deformation. It is widely accepted that Orowan strengthening is not significant for metal matrix composites because the reinforcements are coarse and the interfibre spacing is large. An increase of the yield stress due to the Orowan mechanisms is only some units of $\mathrm{MPa}$.

The value obtained when all the contributions due to the presence of fibres are added to the yield stress of the matrix is very close to the yield stress of the composite observed experimentally. The sum of all individual contributions to strengthening for AZ91 composite with the parallel orientation of fibres gives $329 \mathrm{MPa}$, which is in good agreement with the experimental value for the parallel composite of $324 \mathrm{MPa}$. The main contributions to strengthening are the load transfer and increased dislocation density. Different situation is in the case of WE54+SiC composite. The main contribution to the strengthening is the small grain of the powder metallurgical material i.e. the Hall-Petch strengthening. The equiaxial relatively big particles contribute only lightly to the load transfer and the increased dislocation density. For the yield stress of the WE54+SiC composite at room temperature one obtains 278 $\mathrm{MPa}$ as compared to the experimental value of $246 \mathrm{MPa}$

\subsection{Strain hardening}

The testing temperature affects the shape of the stress-strain curves of $\mathrm{Mg}$ based composites. The strain hardening rapidly decreases with temperature. At high temperatures (above about $200-250^{\circ} \mathrm{C}$ ), a steady state flow (little or no hardening) is attained after small strains. The elongation to fracture of composites increases with increasing temperature. But the composites exhibit lower ductility in comparison to monolithic alloys. The values of the elongation to fracture of the composites are almost an order of magnitude lower that those of the alloys. The lower ductility of composites can be attributed to void nucleation at the matrix-fibre interfaces.

The strain hardening rate is determined by the hardening and softening processes occurring during straining. Plastic deformation is a result of the movement, multiplication and annihilation of dislocations. The main obstacles for glide of dislocations are solute atoms, precipitates, grain boundaries, fibres and dislocations. Plastic zones are formed around fibres (particles). The dislocation density in the vicinity of fibres is higher than elsewhere in the matrix. Moving dislocations cannot pass through the fibres Dislocation pile-ups are formed at fibres and grain boundaries and they can act as stress concentrators. A reduction of the free path of dislocations leads to hardening. On the other hand, the dislocation pile-ups (as stress concentrators) make possible that screw dislocation components may locally cross slip and after the cross slip, annihilation of dislocations may occur. With an increase in the testing temperature, double cross slip is easier leading to a decrease in the strain hardening rate and to an increase in the elongation to fracture, which is in agreement with experimental results. At higher temperatures the coarsening of precipitates may occur during the test. This leads to a reduction to the stress for the dislocation motion and therefore, to a decrease in the strain hardening rate. Annihilation of dislocation is the main softening mechanism.

The primary (dominant) deformation mechanism in Mg alloys is the basal slip. However, five independent slip systems are required for homogeneous deformation of polycrystals [11]. Hence, the activity of non-basal slip systems is required. For magnesium and its alloys, the critical resolved shear stresses (CRSS) of non-basal slip systems are much higher than for basal slip system at room temperature. The CRSS for non-basal slip systems decrease very rapidly with increasing temperature. It means the activity of nonbasal slip systems increases with increasing temperature. In magnesium and its alloys, the glide of $\langle c+a\rangle$ dislocations in second-order pyramidal slip system is expected [12,13]. Intensive activity of non-basal slip systems with $\langle c+a\rangle$ dislocations at higher temperatures provides explanation for the observed decrease of the flow stress and of strain hardening rate. During deformation the motion of dislocations in both the basal and the non-basal slip systems is assumed. The activity of the non-basal slip system increases with increasing temperature. The interaction between basal and non-basal dislocations may result to new obstacles or annihilation depending on the dislocation reaction. Dislocation reactions may produce sessile dislocation and an increase in the density of the forest dislocations. Then, additional obstacles for the dislocation movement are formed, which causes hardening. With increasing temperature, $\langle a\rangle$ dislocations may cross slip from the basal slip system through prismatic or first-order pyramidal planes to parallel basal planes, which causes an increase in the free path of dislocations and therefore softening. Screw components of $\langle c+a\rangle$ dislocations can also move to the parallel slip planes by double cross slip. Annihilation of dislocations can follow after cross slip. It means the density of dislocations decreases, the work hardening rate decreases and softening occurs. It is clear that both the glide of $\langle c+a\rangle$ dislocations on the second-order pyramidal slip planes and cross slip of $\langle a\rangle$ and $\langle c+a\rangle$ dislocation types are strongly thermally activated.

The observed increase in the elongation to fracture with increasing temperature may be also explained by increasing activity of non-basal slip systems with increasing temperature.

\section{Conclusions}

Ceramics fibres and particles influence significantly mechanical properties of composites with the AZ91 and WE54 matrix. Contribution of short fibres to strengthening of the composite is 
higher than particles. This reinforcing phases impact decreases with increasing temperature. The main hardening mechanism in the AZ91 composite is probably the load transfer in which the part of the external load within the matrix is transferred to reinforcement. An increase in the dislocation density and residual thermal stresses play also important role.

The most important contributions to the yield stress in WE54+SiC composite are the small grain size of the matrix alloy and the stress increase due to precipitation hardening in the matrix. The reinforcing phase contributes to strengthening mainly through an increased dislocation density (arising from the thermal and geometrical mismatch). The load transfer from matrix to reinforcing particulates plays in this composite only a marginal role.

The test temperature influences significantly the deformation behaviour of Mg-based composites. The yield strength and the maximum flow stress decrease very rapidly with increasing temperature. The difference between the maximum flow stress and the yield stress decreases with increasing temperature. Therefore, the work hardening rate decreases also with an increase in the test temperature. From the dislocation theory point of view, it means that at higher temperatures there is a dynamic balance between hardening and softening processes. Hardening is a result of storage of dislocations and softening is caused by annihilation of dislocations. The activity of non-basal slip systems plays an important role in the deformation behaviour of magnesium based composites. The glide of $\langle c+a\rangle$ dislocations on the second order pyramidal slip planes and double cross slip of both $\langle a\rangle$ and $\langle c+a\rangle$ dislocations affect significantly the deformation behaviour of magnesium based composites.

\section{Acknowledgements}

The authors acknowledge financial support of the Grant Agency of the Czech Republic under grant 106/06/1354. The authors are grateful also for the support offered by the Czech and Slovak authorities under the Exchange Programme MEB 0808116.

\section{References}

[1] CLYNE, T.W, WITHERS, P.J.: An Introduction to Metal Matrix Composites, Cambridge, Cambridge Univ, Press 1993.

[2] AIKIN Jr, R.M., CHRISTODOULOU, L.: Scripta Metall. Mater., Vol. 25, 1991, p. 9.

[3] CHAWLA, K.K.: In: Materials Science and Technology, Vol. 13, Eds. Cahn R.W., Haasen P., Kramer E.J., Weinheim: VCH, 1993.

[4] URRETA, S.E., SCHALLER, R., CARENO-MORELLI, E., GABELLA, E.J.: Physique IV, 1996, p. C8-774.

[5] DELANNAY. F.: In: Comprehensive Composite Materials, Vol. 3, Ed. Clyne, T.W. Amsterdam, Elsevier, 2000, p. 341.

[6] DUNAND, D.C., MORTENSEN, A.: Acta Metall. Mater., Vol. 39, 1991, p. 127.

[7] ARSENAULT, J.R., SHI, N.: Mater. Sci. Eng., Vol. 81, 1986, p. 151.

[8] ARMSTRONG, R.W.: Acta Metall., Vol. 16, 1968, p. 347.

[9] ASHBY. M.F.: Phil. Mag., Vol. 21, 1970, p. 399.

[10] SCATTERGOOD. R.O., BACON. D.: Phil. Mag. A, Vol. 31, 1975, p. 179.

[11] TAYLOR, G.I: J. Inst. Met., Vol. 62, 1938, p. 307.

[12] LUKAC, P.: Czech. J. Phys. B, Vol. 31, 1981, p. 135.

[13] BALIK, J., LUKAC, P., BOHLEN, J., KAINER, K.U.: Metallic Materials, Bratislava, Vol. 45, 2007, p. 135. 